\title{
Impact of Climate Drift on Twenty-First-Century Projection in a Coupled Atmospheric-Ocean General Circulation Model
}

\author{
MAO-CHANG LIANG \\ Research Center for Environmental Changes, and Institute of Astronomy and Astrophysics, Academia Sinica, Taipei, \\ and Graduate Institute of Astronomy, National Central University, Zhongli City, Taiwan \\ LI-CHING LIN \\ Institute of Earth Sciences, Academia Sinica, Taipei, Taiwan \\ KA-KIT TUNG \\ Department of Applied Mathematics, University of Washington, Seattle, Washington \\ YUK L. YUNG \\ Division of Geological and Planetary Sciences, California Institute of Technology, Pasadena, California \\ SHAN SUN \\ Earth System Research Laboratory, National Oceanic and Atmospheric Administration, Boulder, Colorado
}

(Manuscript received 9 May 2013, in final form 25 June 2013)

\begin{abstract}
Reducing climate drift in coupled atmosphere-ocean general circulation models (AOGCMs) usually requires 1000-2000 years of spinup, which has not been practical for every modeling group to do. For the purpose of evaluating the impact of climate drift, the authors have performed a multimillennium-long control run of the Goddard Institute for Space Studies model (GISS-EH) AOGCM and produced different twentiethcentury historical simulations and subsequent twenty-first-century projections by branching off the control run at various stages of equilibration. The control run for this model is considered at quasi equilibration after a 1200-yr spinup from a cold start. The simulations that branched off different points after 1200 years are robust, in the sense that their ensemble means all produce the same future projection of warming, both in the global mean and in spatial detail. These robust projections differ from the one that was originally submitted to the Intergovernmental Panel on Climate Change (IPCC) Fourth Assessment Report (AR4), which branched off a not-yet-equilibrated control run. The authors test various common postprocessing schemes in removing climate drift caused by a not-yet-equilibrated ocean initial state and find them to be ineffective, judging by the fact that they differ from each other and from the robust results that branched off an equilibrated control. The authors' results suggest that robust twenty-first-century projections of the forced response can be achieved by running climate simulations from an equilibrated ocean state, because memory of the different initial ocean state is lost in about 40 years if the forced run is started from a quasi-equilibrated state.
\end{abstract}

\section{Introduction}

Global temperature is likely to increase further if greenhouse gas emissions continue unchecked (Brohan

Corresponding author address: Mao-Chang Liang, Research Center for Environmental Changes, Academia Sinica, No. 128, Academic Road, Taipei 115, Taiwan.

E-mail: mcl@rcec.sinica.edu.tw et al. 2006; Hansen et al. 2001, 2006; Smith et al. 2008; Pachauri and Reisinger 2007; Broecker 2007). Various mitigation efforts are being negotiated and policies implemented to keep the increase under, for example, $2^{\circ} \mathrm{C}$ (Meinshausen et al. 2009). Such policy efforts are informed by projections of coupled atmosphere-ocean general circulation models (AOGCMs).

Since the Third Assessment Report of the Intergovernmental Panel on Climate Change (IPCC), flux adjustment 
has been abandoned by most AOGCMs. Instead, these models rely on the control runs having run long enough to attain quasi equilibration between the atmosphere and oceans before the twentieth-century historical simulation is carried out. However, it usually takes more than 1000 years for a model control to attain such a quasiequilibrated state in the absence of forcing trends. In practice, twentieth-century simulations were commonly performed after a few hundred years of spinup. It can be shown that most Coupled Model Intercomparison Project 3 (CMIP3) (Meehl et al. 2007) models have serious climate drift problems (Gupta et al. 2012). The consequences of the climate drifts are most problematic for multidecadal- to century-long projections-an important range for policy decisions. For longer-range projections under increasing greenhouse forcing, the forced solution overwhelms the effect of climate drifts.

In very long control runs (called "control" here) without forcing trends, there are three stages of climate drift; only the first two stages are commonly known as "climate drift." The first is the "coupling shock" (also called the "major drift")- the large but rapid change when the atmosphere and ocean modules are first coupled-and "millennium drift"- the slow drift that takes several hundred years. No models participating in IPCC Fourth Assessment Report (AR4) (Solomon et al. 2007) submitted runs that branched off control runs beyond the millennium drift stage (Gupta et al. 2012). Remedies adopted in AR4 consist mainly of subtracting the "drift" from a parallel control (with preindustrial conditions) from the twentiethcentury simulation and subsequent twenty-first-century projection. Since the process governing ocean heat uptake is highly nonlinear, subtracting either the surface temperature or the heat flux of the parallel control from the simulation may not be the correct solution. There is additionally a third stage, "the quasi equilibration" in very long control runs, which, despite its name, still contains large low-frequency variability. Some such variability has multicentury-long periods and behaves like the second stage of climate drift. In some other models the internal variability has such large amplitudes that it affects the initial state used depending on when the twentiethcentury simulation branches off the control.

We have performed a multimillennium-long integration using the Goddard Institute for Space Studies model (GISS-EH; Shindell et al. 2006; Sun and Bleck 2006) (the model is a slightly updated version that GISS-EH used for IPCC AR4 to correct a minor error in ozone). In the preindustrial control, the amount of greenhouse gases and atmospheric aerosol loading is kept at a constant preindustrial level. For validation purposes (Camp and Tung 2007; Tung et al. 2008), solar forcing varies periodically in an 11-yr solar cycle.

\section{Control runs}

Long-term integrations of the control are shown in Fig. 1, starting with 1850 atmospheric conditions. For GISS-EH, there was no spinup done prior to the start of the control (Gupta et al. 2012; Sun and Bleck 2006). The large $0.5^{\circ} \mathrm{C}$ drop during first 20 years (the coupling shock) in Fig. 1 is followed by a significant secular cooling of $0.5^{\circ} \mathrm{C}$ for the next 350 years (Shindell et al. 2006). [The twentieth-century simulation was done for AR4 off the control 200 years after the start, as indicated by the red arrow in Fig. 1. This branching-off point was chosen by the GISS team (Shindell et al. 2006).] The model control then takes 800 years to warm, and is considered to be "quasi equilibrated" 1200 years after the start (see also Liang et al. 2013), although the presence of 500-yr lowfrequency variability in this model makes such a determination somewhat arbitrary. No scenario or attribution runs should have been done by branching off the first 1200 years of GISS-EH control.

\section{Twentieth-century climate simulations and twenty-first-century projections}

It is widely accepted that climate simulations are sensitive to initial conditions and to changing computer hardware. An ensemble average is often used to remove the chaos and reveal the forced solution (Deser et al. 2012). What we are addressing here is a different problem: because the longer-term drift is approximately common among the ensemble members, it cannot be removed by common ensemble averaging. In projecting future warming under emission scenarios, it is understood that models do not necessarily produce the correct internal variability and so ensemble averaging is commonly used to reveal the forced response as different phases of model internal variability, such as El Niño or Atlantic multidecadal oscillation, tend to average out. However, since climate drift is an unforced variability that remains after ensemble averaging, the interpretation of the ensemble mean as the forced response is correct only if model drift can be removed.

We produce the ensemble mean (with five ensemble members each initiated a decade apart) of the twentiethcentury historical simulation from 1850 to 2005 using the same changing radiative forcing and the same initial conditions as those submitted to the CMIP3. The runs are then continued to 2100 under the AR4 A1B emission scenario (Pachauri and Reisinger 2007). In Fig. 2, we show the spatial pattern of the projected warming at calendar years 2050 and 2090. We take 20-yr averages centered at these two years. No postprocessing, such as subtracting out the parallel control climate drift, is done. Run A branched off 2240 years after the start and run B 

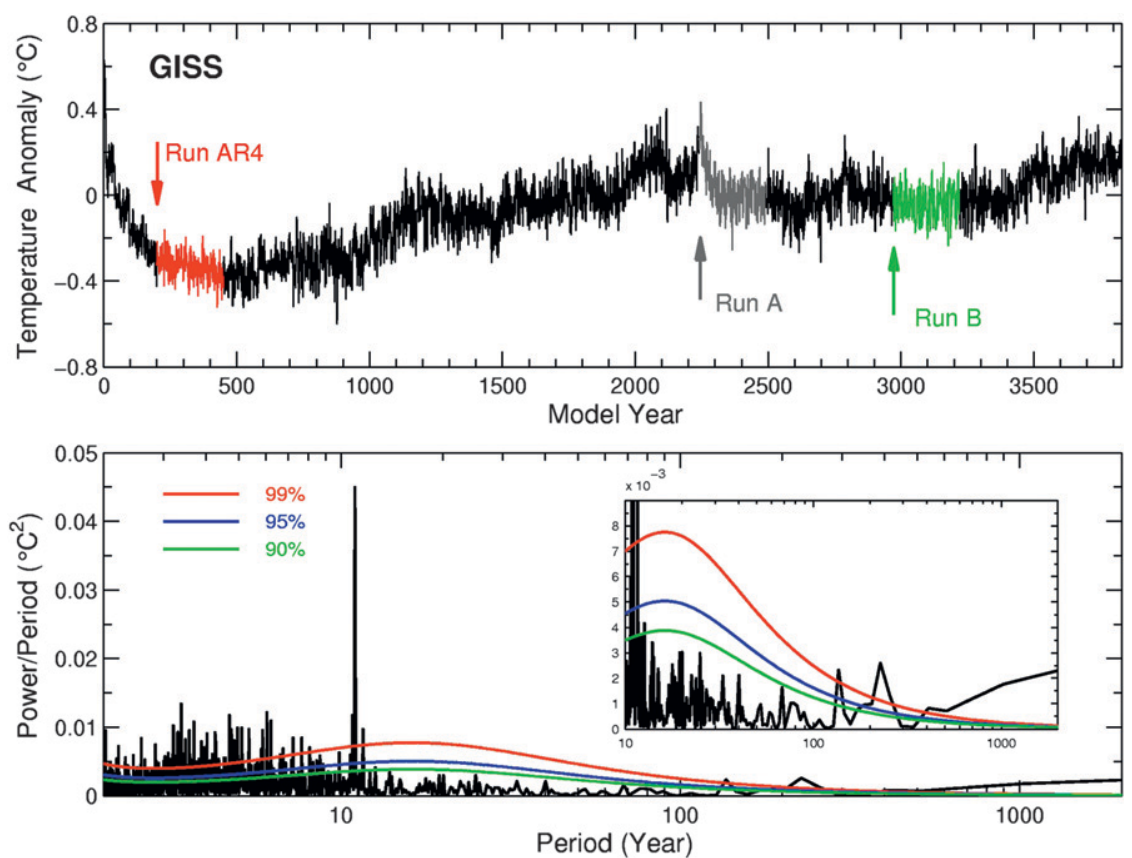

FIG. 1. (top) Global-mean surface air temperature for GISS-EH from a multimillennium preindustrial control experiment with no changes in anthropogenic forcing, and (bottom) its Fourier spectrum (only quasi-equilibrium states are considered). Confidence levels at $99 \%$, $95 \%$, and $90 \%$ are shown by the red, blue, and green curves, respectively. The three branch-off points referred to later in the paper are marked by arrows, and the underlying climate drift of each simulation run is shown by the corresponding color.

branched off 2970 years after the start of the control. The projection is robust in spatial details as long as it is produced off an equilibrated control, despite the fact that one branching-off point gives a warm initial condition and one a cold initial condition. In fact, the initial conditions for runs $\mathrm{A}$ and $\mathrm{B}$, indicated by the arrows in the top panel of Fig. 1, were chosen to highlight the difference in the initial conditions. This robust behavior is in contrast to the projection of the original run submitted to AR4 (labeled "AR4 run"), with a not-yet-equilibrated branching-off point from the control. The qualitative features are especially different in the multidecadal (year 2050) projections. The run denoted as AR4 misses the North Atlantic cooling. Instead the cooling occurs in the North Pacific. The Arctic amplification of warming is only over the northeastern part of Canada. This figure demonstrates the sensitivity of the 50-yr projected warming to the presence or absence of climate drift. It also shows that when the runs branch off equilibrated control, the solution is insensitive to the initial condition, which is quite different, as shown in the top panels of Fig. 2, and yet the eventual warming is almost the same.

It is often thought that the effect of climate drift can be removed (or reduced) if we subtract the drift of the parallel control from the simulation and projection runs.
The effect is shown in Fig. 3 with different offsets, all with the subtraction of the corresponding control trend, as in AR4. In the top panels, each run is offset by its initial condition, so that what is shown is the warming anomaly from 1850 . The subtraction of the climate drift in control not only does not reduce the difference of the not-yet-equilibrated run from the equilibrated runs, but it introduces artificial differences between the equilibrated runs that were not in the raw model output. Amplified warming in the Arctic reported previously for this model is not found when started from the equilibrated control, while Antarctic warming is much more amplified. This is caused by the subtraction of the initial state in defining the "warming anomaly." Nevertheless, the feature of amplified polar warming emerges in both poles by year 2090. By then, the forced solution dominates over the climate drift. For presentation purposes in AR4's chapter 10, each model's 1980-99 mean is subtracted; this is shown in the bottom panels of Fig. 3 . Almost all prominent continental and oceanic features are different by 2050 in the three runs. The conclusion is that the subtraction of the parallel control drift from the simulation runs cannot remedy the drift problem and in addition creates an artificial difference between equilibrated runs that were not in the raw model output. 


\section{Initial states}

Run AR4

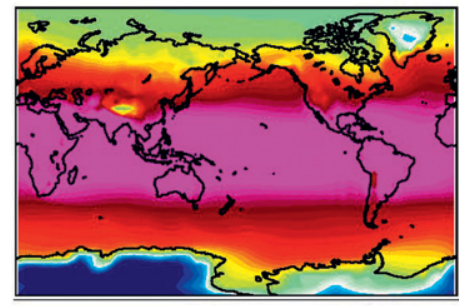

Run A

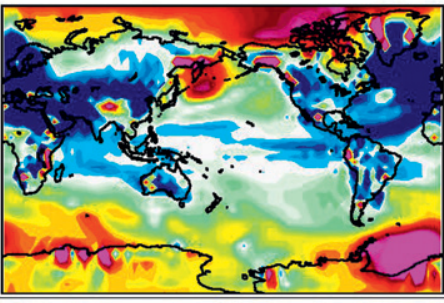

A1B projection
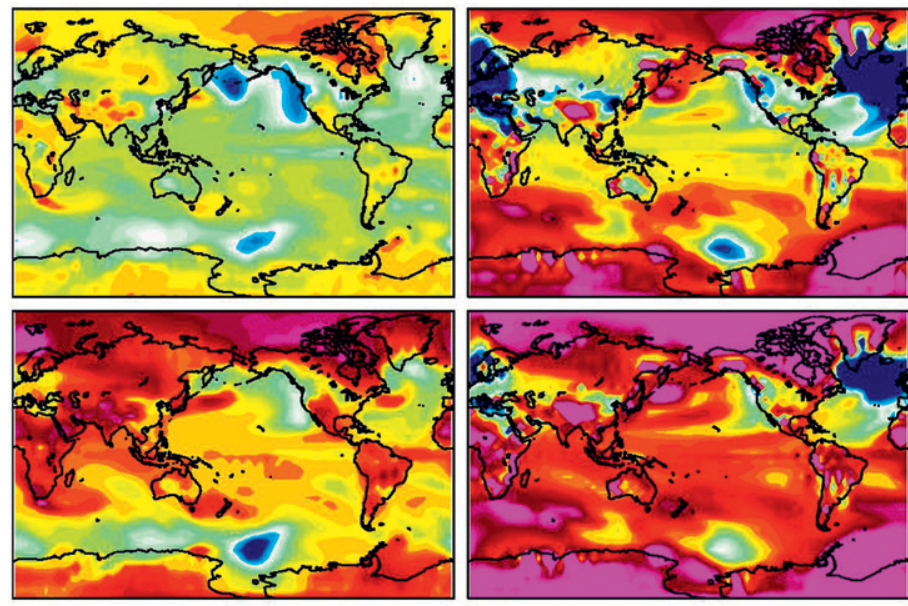

Run B
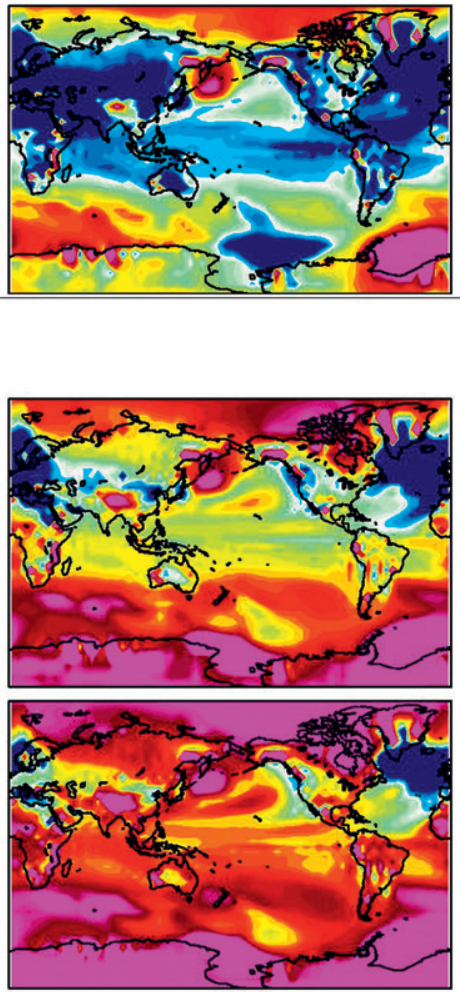

$\mathrm{T} /{ }^{\circ} \mathrm{C}$

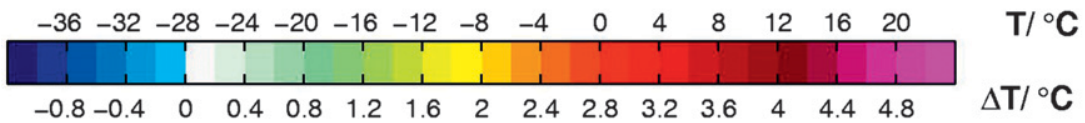

FIG. 2. Ensemble-mean surface air temperature for the A1B emission scenario run as a continuation of the twentieth-century historical simulation. (top) Annually averaged GISS-EH initial state (year 1850) processed as in AR4, and two other runs, A and B, from a fully adjusted ocean (only the difference from the GISS-EH initial state is shown; bottom color scale). The 20-yr-averaged patterns centered at years (middle) 2050 and (bottom) 2090 are shown for the projections. Only a common pattern (the initial condition of the AR4 run) is subtracted, which is done for better visualization only.

Therefore, this commonly adopted remedy for climate drift is not recommended.

Figure 4 compares the global-mean, ensemble-mean surface air temperature of the three runs for the period 1850-2100. The red curve reproduces the AR4 GISSEH model result simulated 200 years after the start of the control. The green and gray curves represent runs that branch off "quasi equilibrated" states in the control. In Fig. 4a, the "raw" results are shown without postprocessing. All runs off the quasi-equilibrated control converge to each other after 40 years. Afterward the difference from these runs is surprisingly small. This fact has been verified with 20 individual runs of GISS-EH and also confirmed using the Community Climate System Model, version 4 (CCSM4). This was shown in Liang et al.
2013 using the same $1 \% \mathrm{yr}^{-1}$ increase in $\mathrm{CO}_{2}$. The projection is robust as long as the model is adequately spun up. In the rest of Fig. 4, common remedies to deal with climate drift are applied. In Fig. 4b, the parallel control is subtracted year by year. In Figs. $4 c$ and $4 d$, the linear trend of the parallel control is subtracted, but with different offsets. In Figs. $4 \mathrm{~b}$ and $4 \mathrm{c}$, the projected warming by the equilibrated runs $\mathrm{A}$ and $\mathrm{B}$ is the same regardless of whether the actual control or its linear trend is subtracted, but they both are warmer after the mid-twentyfirst century, by about $0.6^{\circ} \mathrm{C}$, than the original GISS-EH projection, which branched off a not-yet-equilibrated control. To put that difference in perspective, runs A and $\mathrm{B}$ now project that the $2^{\circ} \mathrm{C}$ warming threshold from preindustrial conditions will be crossed a decade before 


\section{Offset to 1850}

Run AR4
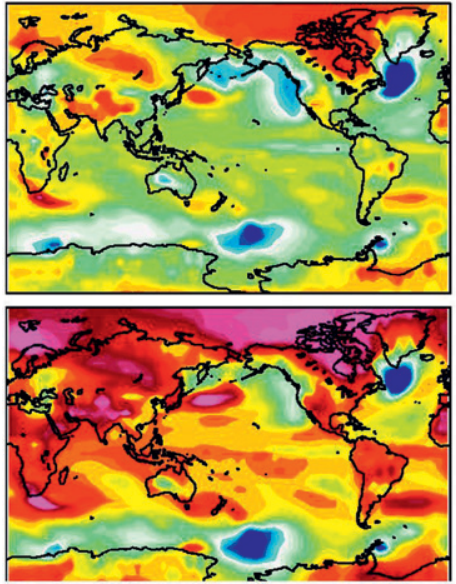

Run A
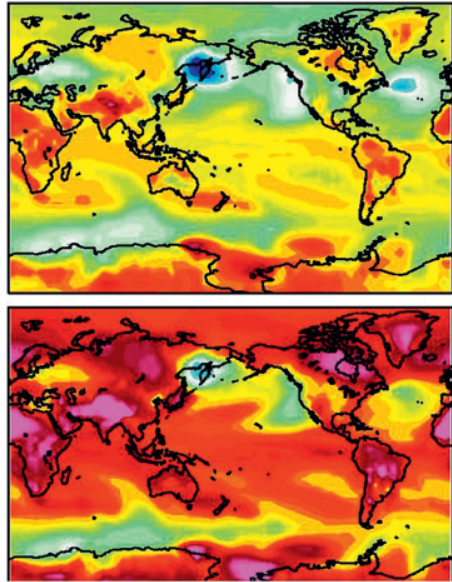

Offset to 1980-1999
Run B
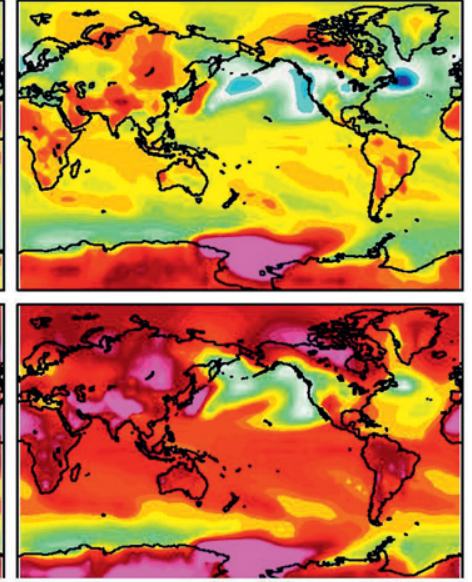
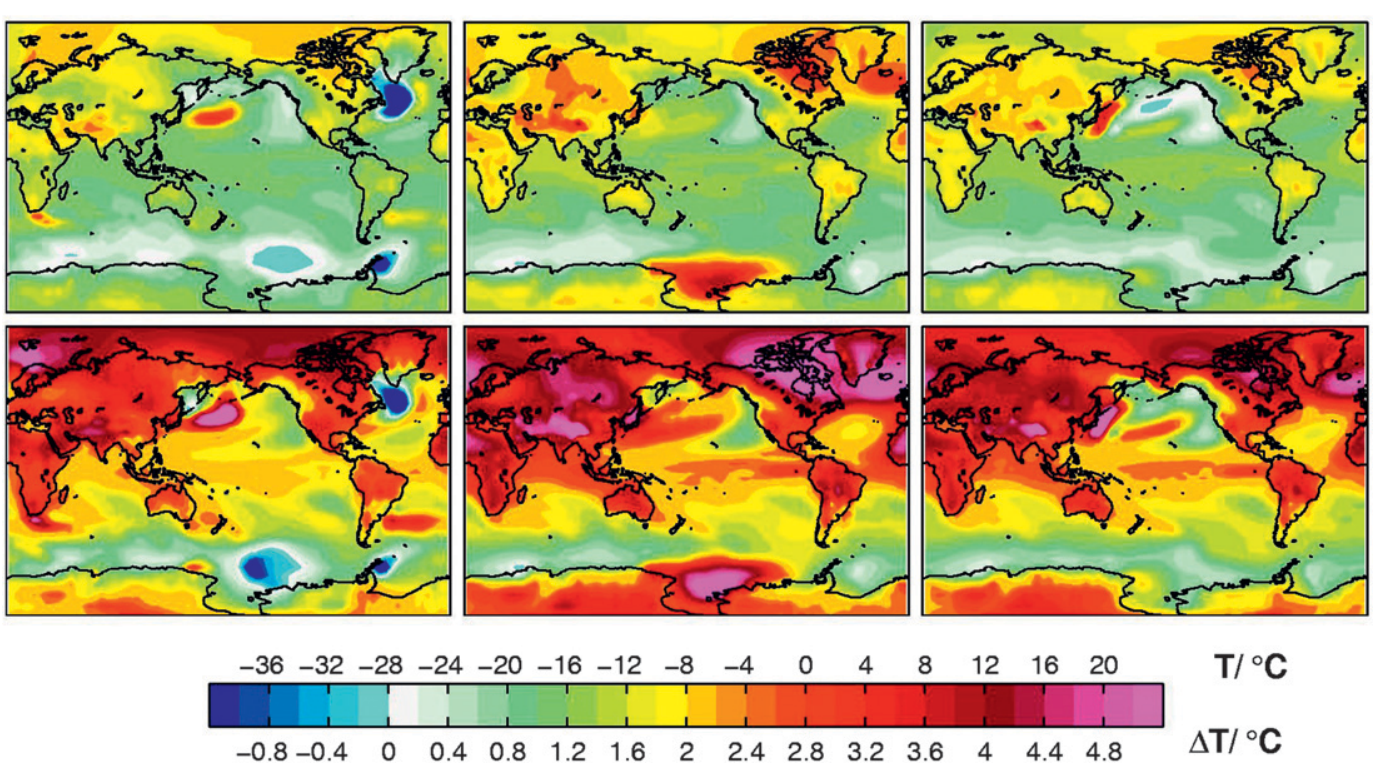

$\mathrm{T} /{ }^{\circ} \mathrm{C}$

$\Delta \mathbf{T} /{ }^{\circ} \mathrm{C}$

FIG. 3. As in Fig. 2, but (top) with the subtraction of the corresponding control run trend and then offset to each run's 1850 initial state. (bottom) The offset used is each run's 1980-99 mean climatology.

that projected by GISS-EH for AR4. This highlights the importance of dealing with the climate drift for such projections.

Intermodel difference was reduced in the AR4 presentation by subtracting from each model result its own mean in a certain period-say 1951-80. This is shown in Fig. $4 \mathrm{~d}$ for the same three simulations. Obviously the difference between all runs is reduced not only for 195180 but also for a decade and half after. However, there is still a $0.5^{\circ} \mathrm{C}$ difference in 2099 between the gray (run A branching off equilibrated control) and the red curve (branching off nonequilibrated control). All historical simulations agree remarkably well with the observation, similarly offset, and so that good agreement cannot be used to select a "best" run for the projection to the future.

\section{Similar behavior in another AOGCM}

Are the results reported above peculiar to the GISSEH AOGCM? To find out, we have carried out a 5000-yr control run of CCSM4 (North et al. 2011) at T31 resolution (Liang et al. 2013, their Fig. 1). CCSM4 is the model being used for the forthcoming assessment report. There were some differences in the forcing among models participating in AR4, which make detailed intermodel comparison difficult. In that paper, Liang et al. (2013) 


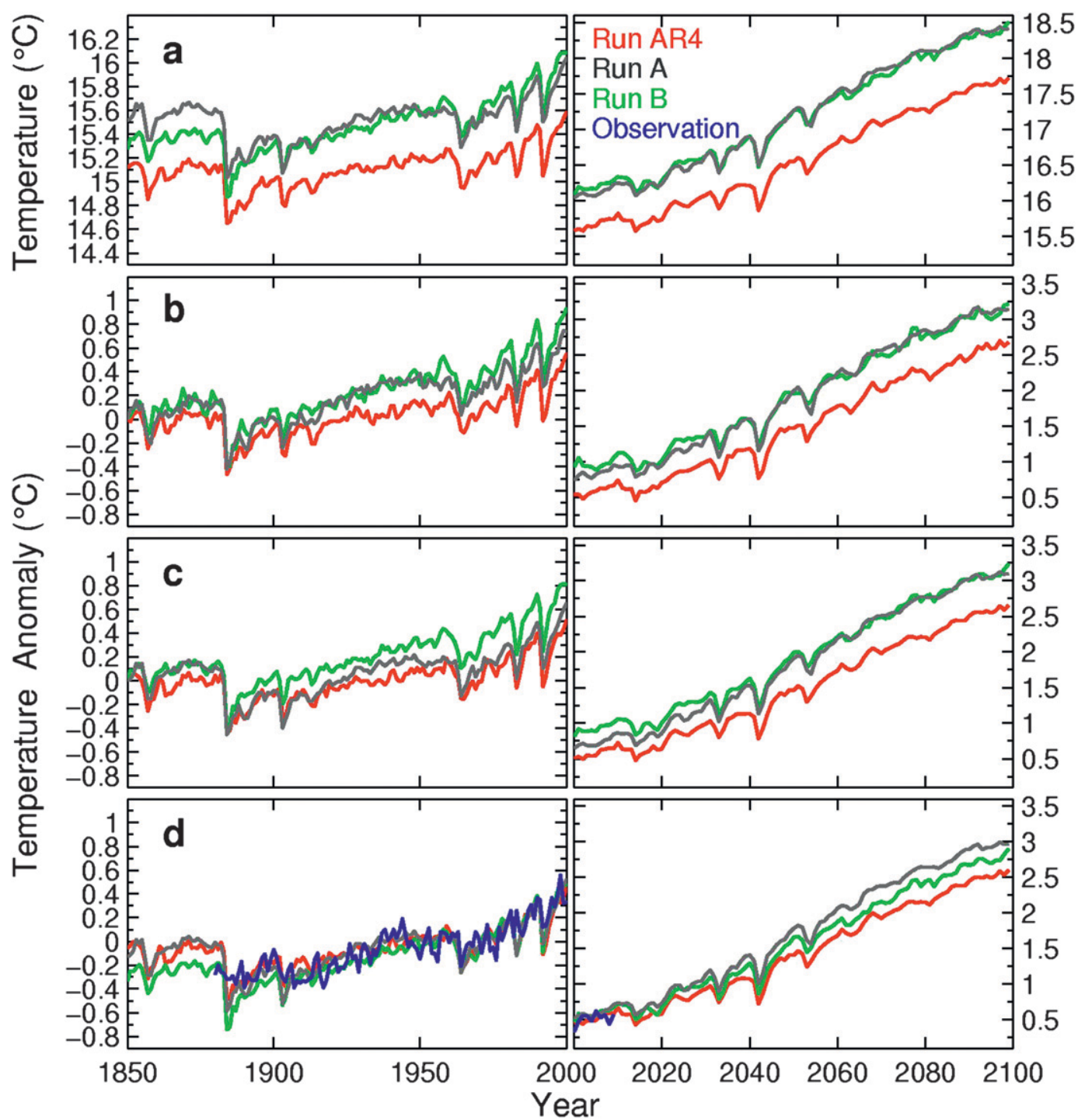

FIG. 4. Ensemble mean of globally and annually averaged surface air temperature for (left) historical simulation runs and (right) future A1B scenario runs. Each ensemble member is generated by initiating at different 10-yr intervals centered at 2240 years after the start of the preindustrial control (gray) and at 2970 years after the start (green), along with simulations published online by GISS (run AR4; red) to correct for errors made in the version submitted to AR4, which branched off 200 years after the start of the control. (a) Surface air temperature derived from the model output. (b) Warming anomaly, relative to each run's 1850 initial condition, with year-to-year control run subtraction. (c) Following the IPCC protocol, the underlying linear trend of the control is removed, in addition to offsetting each run to zero at 1850. (d) Each model run's 1951-80 mean is subtracted for presentation, in addition to the subtraction of the control trend. The observation curve (blue) is the deviation from the value averaged over years 1951-80, as presented in AR4 (Hansen et al. 2010). Note the changes in scale in the right panels, which are simply continuations of the corresponding left panels.

reported the results of runs, each 80-yr long, for each of the two models (CCSM4 and GISS-EH) using the same $1 \% \mathrm{yr}^{-1}$ increase in $\mathrm{CO}_{2}$ in radiative forcing, by branching off over 50 different points in the control runs. These runs convinced us that the results reported here are common to the two models, despite their different time trajectories of spinup shown in their respective preindustrial control runs.

\section{Conclusions}

We report an underappreciated uncertainty in transient climate simulations: Simply by running an AOGCM longer in a control run before we branch off the control and start the climate simulation, the projected future warming changes. This is true even if the drift in the control run is subtracted out. The culprit is the so-called 
climate drift, caused by the imbalance of the model ocean state with its atmosphere. Subtraction of the contemporaneous control run, a commonly adopted remedy, cannot remove the climate drift resulting from such an imbalance.

The problem discussed here is different from that of decadal predictability. On decadal time scales, the difference shown among different runs is due mainly to the difference in the initial conditions (see Fig. 2, top, and Fig. 4a), which exists even at quasi equilibrium. It is well known that decadal prediction is highly dependent on the memory of the initial condition (Meehl et al. 2009). While ensemble averaging reduces the impact of the initial conditions in favor of the forced response, the effect of climate drifts still appears in the ensemble mean. Nevertheless, we have shown that, provided that the initial state for both the atmosphere and the oceans is derived from a quasi-equilibrium control, the memory of the initial condition is lost after 40 years. Beyond that, the ensemble mean solution is controlled by the trajectory of the radiative forcing, such as the change in greenhouse gases. While unforced internal variability is often substantial in the control run, such variability is not carried over to the ensemble-mean simulation after 40 years. Robust projections can be achieved under moderate to strong forcing, but only if the model has been adequately spun up.

Based on our findings, we propose that all models participating in future assessment reports should have been run long enough for the control to be beyond the "millennium drift" stage. By doing so, a robust future climate projection can be made as the forced response to the changing radiative forcing without further subtraction of the contemporaneous control run drift.

Acknowledgments. We thank editor Ming Cai for his detailed suggestions and two anonymous reviewers for their comments that helped focus the presentation. This work is supported in part by NSC Grant 101-2628-M001-001-MY4 to Academia Sinica and its Grid Computing Center, KKT's research by NSF Grants ATM 0808375 and DMS 0940342, and YLY's by Caltech's KISS program.

\section{REFERENCES}

Broecker, W. S., 2007: Climate change: $\mathrm{CO}_{2}$ arithmetic. Science, 315, 1371-1371.

Brohan, P., J. J. Kennedy, I. Harris, S. F. B. Tett, and P. D. Jones, 2006: Uncertainty estimates in regional and global observed temperature changes: A new data set from 1850. J. Geophys. Res., 111, D12106, doi:10.1029/2005JD006548.

Camp, C. D., and K. K. Tung, 2007: Surface warming by the solar cycle as revealed by the composite mean difference projection. Geophys. Res. Lett., 34, L14703, doi:10.1029/2007GL030207.

Deser, C., A. Phillips, V. Bourdette, and H. Teng, 2012: Uncertainty in climate change projections: The role of internal variability. Climate Dyn., 38, 527-546.

Gupta, A. S., L. C. Muir, J. N. Brown, S. J. Phipps, P. J. Durack, D. Monselesan, and S. E. Wijffels, 2012: Climate drift in the CMIP3 models. J. Climate, 25, 4621-4639.

Hansen, J., R. Ruedy, M. Sato, M. Imhoff, W. Lawrence, D. Easterling, T. Peterson, and T. Karl, 2001: A closer look at United States and global surface temperature change. J. Geophys. Res., 106 (D20), 23 947-23963.

, M. Sato, R. Ruedy, K. Lo, D. W. Lea, and M. Medina-Elizade, 2006: Global temperature change. Proc. Natl. Acad. Sci. USA, 103, 14288-14293.

- R. Ruedy, M. Sato, and K. Lo, 2010: Global surface temperature change. Rev. Geophys., 48, RG4004, doi:10.1029/ 2010RG000345.

Liang, M. C., L. C. Lin, K. K. Tung, Y. L. Yung, and S. Sun, 2013: Transient climate response in coupled atmosphericocean general circulation models. J. Atmos. Sci., 70, 12911296.

Meehl, G. A., C. Covey, T. Delworth, M. Latif, B. McAvaney, J. F. B. Mitchell, R. J. Stouffer, and K. E. Taylor, 2007: The WCRP CMIP3 multimodel dataset: A new era in climate change research. Bull. Amer. Meteor. Soc., 88, 1383-1394.

, and Coauthors, 2009: Decadal prediction. Bull. Amer. Meteor. Soc., 90, 1467-1485.

Meinshausen, M., N. Meinshausen, W. Hare, S. C. B. Raper, K. Frieler, R. Knutti, D. J. Frame, and M. R. Allen, 2009: Greenhouse-gas emission targets for limiting global warming to $2^{\circ} \mathrm{C}$. Nature, $\mathbf{4 5 8}, 1158-1162$.

North, G. R., J. Wang, and M. G. Genton, 2011: Correlation models for temperature fields. J. Climate, 24, 5850-5862.

Pachauri, R. K., and A. Reisinger, Eds., 2007: Climate Change 2007: Synthesis Report. Cambridge University Press, 104 pp.

Shindell, D., G. Faluvegi, A. J. Miller, G. A. Schmidt, J. Hansen, and S. Sun, 2006: Solar and anthropogenic forcing of tropical hydrology. Geophys. Res. Lett., 33, L24706, doi:10.1029/ 2006GL027468.

Smith, T. M., R. W. Reynolds, T. C. Peterson, and J. Lawrimore, 2008: Improvements to NOAA's Historical Merged LandOcean Surface Temperature analysis (1880-2006). J. Climate, 21, 2283-2296.

Solomon, S., D. Qin, M. Manning, Z. Chen, M. Marquis, K. Averyt, M. M. B. Tignor, and H. L. Miller Jr., Eds., 2007: Climate Change 2007: The Physical Science Basis. Cambridge University Press, 996 pp.

Sun, S., and R. Bleck, 2006: Multi-century simulations with the coupled GISS-HYCOM climate model: Control experiments. Climate Dyn., 26, 407-428.

Tung, K. K., J. S. Zhou, and C. D. Camp, 2008: Constraining model transient climate response using independent observations of solar-cycle forcing and response. Geophys. Res. Lett., 35, L17707, doi:10.1029/2008GL034240. 